\title{
Keratocyte Apoptosis Associated with Keratoconus
}

\author{
WOO-JUNG KIM ${ }^{\text {ac }}$, YARON S. RABINOWITZ ${ }^{\text {d }}$ DAVID M. MEISLER \\ AND STEVEN E. WILSON ${ }^{\mathrm{bc} *}$ \\ ${ }^{a}$ Department of Ophthalmology, Sungkyunkwan University School of Medicine, Seoul, Korea, \\ ${ }^{b}$ Department of Ophthalmology, University of Washington School of Medicine, Seattle, WA 98195-6485, \\ U.S.A., ${ }^{c}$ Eye Institute, The Cleveland Clinic Foundation, and ${ }^{d}$ The Cornea-Genetic Eye Institute, \\ Cedars-Sinai Medical Center, Los Angeles, CA, U.S.A.
}

(Received Cleveland 18 January 1999 and accepted in revised form 18 May 1999)

\begin{abstract}
Keratoconus is an ectatic corneal dystrophy associated with stromal thinning and disruption of Bowman's layer. The purpose of this study was to explore a possible association between keratocyte apoptosis and keratoconus. Keratocyte apoptosis was evaluated in corneas of patients with keratoconus, corneas of patients with stromal dystrophies, and normal donor corneas using the transferase-mediated dUTP-digoxigenin nick and labeling (TUNEL) assay. Keratocyte apoptosis was also studied in keratoconus and normal corneas using transmission electron microscopy. TUNEL-stained keratocytes were detected in $60 \%$ of corneas with keratoconus, but only $35 \%$ of corneas with stromal dystrophies $(P=0 \cdot 03)$. The number of TUNEL-positive keratocytes detected in the keratoconus, stromal dystrophy, and normal corneas was $7 \pm 1$ (mean \pm standard error, range 0-20), $2 \pm 0 \cdot 8$ (range 0-9), and $0 \pm 0$ (range 0-0) TUNEL-positive cells per section, respectively. The differences between the keratoconus and the stromal dystrophy $(P=0.0097)$ or the normal cornea $(P=0.01)$ groups were statistically significant. The difference between the stromal dystrophy and normal cornea groups was not statistically significant $(P=0.45)$. The stromal dystrophy group was included to account for surgery-associated keratocyte apoptosis. No TUNEL-stained keratocytes were detected in normal corneas. Cell morphologic changes consistent with apoptosis were detected by transmission electron microscopy (TEM) in keratocytes of keratoconus corneas, but not in keratocytes in normal corneas. Chronic keratocyte apoptosis associated with ongoing epithelial injury may link risk factors associated with keratoconus such as chronic eye rubbing, contact lens wear, or atopic eye disease. Similarly, increases that have been detected in several different degradative enzymes in keratoconus corneas could be associated with chronic keratocyte apoptosis and less than perfect control of release of intracellular contents. (C) 1999 Academic Press

Key words: apoptosis; contact lenses; keratocytes; keratoconus; pathogenesis.
\end{abstract}

\section{Introduction}

Keratoconus is the most common ectatic dystrophy of the cornea (Kennedy et al., 1986). The disease is characterized by progressive thinning of the corneal stroma that typically occurs over decades. Bowman's layer is commonly disrupted, especially in advanced cases. Keratoconus leads to corneal steepening associated with myopia and severe irregular astigmatism that limits the vision obtainable with glasses (Wilson and Klyce, 1991; Rabinowitz, 1998). In many cases, vision can be restored with rigid gas-permeable contact lenses, but in some eyes advances to a point that a corneal transplant must be performed (Krachmer et al., 1984).

The pathophysiological processes underlying keratoconus have yet to be elucidated. Clinical studies have suggested that the disease is associated with contact lens wear (Koreman, 1986; Macsai et al., 1990), chronic eye rubbing (Coyle, 1984), and atopy of the eye (Rahi et al., 1977; Harrison et al., 1989).

Previous studies in mice and rabbits have demon-

* Address correspondence to: Steven E. Wilson, Department of Ophthalmology, University of Washington School of Medicine, Box 356485, Seattle, WA 99195-6485, U.S.A. strated that injury to the corneal epithelium is associated with keratocyte apoptosis (Wilson et al., 1996a; Helena et al., 1998). Studies have also demonstrated loss of anterior keratocytes in primates after epithelial injury, although apoptosis has not been conclusively demonstrated in the primate model (Campos et al., 1994). Recent studies have suggested that this response represents mechanical activation of an anti-viral system designated to limit extension of pathogens such as the herpes simplex virus that primarily infect the corneal epithelium, but can spread via infection of adjacent cells to deeper layers of the cornea and into the eye (Wilson et al., 1997). It has been hypothesized that unpropitious and chronic triggers to keratocyte apoptosis may lead to changes in the cornea that include a decrease in the total number of keratocytes, release of degradative enzymes, and loss of corneal stroma over a period of time (Wilson et al., 1996; Wilson and Kim, 1998).

The purpose of this study was to determine whether keratocyte apoptosis was detectable in corneas with keratoconus removed at the time of corneal transplantation. Control studies were performed in corneas with stromal dystrophies and normal corneas removed at autopsy. 


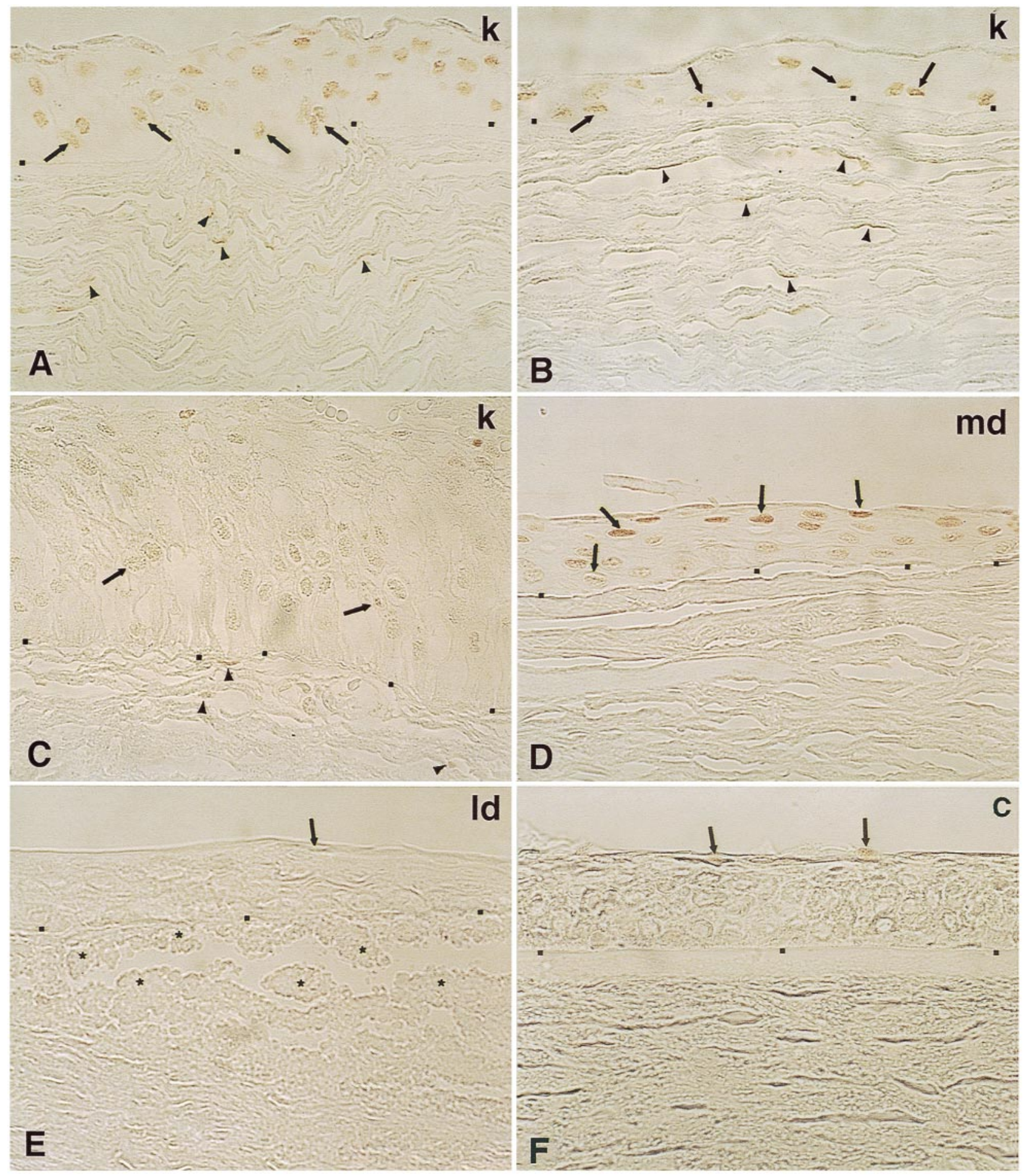

FIG. 1. TUNEL assay detection of apoptosis in representative keratoconus (A-C), stromal dystrophy (D,E), and normal (F) corneas. All images were photographed in the central cornea. Arrowheads indicate TUNEL positive keratocytes. k, md, ld, or $\mathrm{c}$ in upper right hand corner indicates sections from keratoconus, macular dystrophy, lattice dystrophy, or normal corneas, respectively. Small squares indicate the level of the basement membrane of the epithelium. Arrows indicate TUNEL-positive epithelial cells that are a normal part of the maturation of the corneal epithelium. The asterisks in (E) indicate an anterior stromal deposit related to lattice dystrophy. No TUNEL-positive keratocytes were detected in this macular dystrophy (D) or lattice dystrophy (E) cornea. Normal Bowman's layer is present below the epithelial basement membrane (squares) in the normal cornea $(\mathrm{F})$. All panels are at $400 \times$ magnification. 


\section{Materials and Methods}

Peroxidase-based terminal deoxyribonucleotidyl transferase-mediated dUTP-digoxigenin nick and labeling (TUNEL) assay (Wilson et al., 1996) was performed according to the manufacturer's instructions (ApopTag assay, Oncor, Gaithersburg, MD, U.S.A.) to detect fragmentation of DNA associated with apoptosis. Seven micrometer thick sections that extended transversely across the center of the corneal button were cut with a keratome, placed on Superfrost plus microscope slides (Fisher Scientific, Pittsburgh, PA, U.S.A.), and deparafinized for TUNEL staining using standard methods.

A preliminary experiment was performed to study the effectiveness of TUNEL assay on sections of formalin-fixed and paraffin-embedded corneas. We examined 11 recipient corneal buttons removed for central corneal scarring to determine how close to the full-thickness trephine cut keratocyte apoptosis would be detected (from injury to the peripheral epithelium at the time of trephination). No stained keratocyte were detected more than $200 \mu \mathrm{M}$ from the trephine cut (mean distance of farthest TUNEL assay-stained keratocytes from trephine cut $61 \pm 46 \mu \mathrm{m})$. Subsequent observations on keratoconus and control corneas were made at least $500 \mu \mathrm{M}$ from the edge of the recipient button, measured with a reticle to insure that no TUNEL-positive keratocytes detected were due to the peripheral trephine cut at the time of transplantation.

We analysed 45 corneas with clinical and histopathologic keratoconus and 17 corneas with macular, granular, or lattice corneal dystrophy, obtained from the pathology tissue archives at The Cleveland Clinic Foundation, to determine whether TUNEL assaystained keratocytes could be detected. Each corneal block from keratoconus or other corneal dystrophy corneas was obtained from patients who had corneal transplantation at The Cleveland Clinic Foundation by one of two surgeons. Corneal transplant surgery had been performed 6 months to 10 years prior to TUNEL analysis. Two normal, unwounded human corneas (removed for orbital tumor) and eight normal corneas removed within $4 \mathrm{hr}$ of death were also analysed. All normal corneas were also fixed immediately in formalin at the time the recipient cornea was removed from the recipient eye. Following fixation for 2-3 days after removal of the cornea, the buttons were embedded in paraffin. All retrospective tissues were stored as paraffin blocks until sectioning for this study. Keratoconus, corneal dystrophy, and normal corneas were evaluated for keratocyte apoptosis using the TUNEL assay by the identical methods described above.

Full sections across the center of each corneal button were analysed at $400 \times$ magnification for TUNEL-stained keratocytes. TUNEL-positive keratocytes were confirmed at $1000 \times$ magnification. If any TUNEL-stained cells were detected within the cornea of a single section (excluding $500 \mu \mathrm{M}$ at each edge of the button), the cornea was counted as positive. The number of TUNEL-positive keratocytes detected in the section from each keratoconus, other stromal dystrophy, or normal cornea was also recorded.

To confirm cellular morphologic changes consistent with apoptosis, we also examined six keratoconus corneas placed immediately in transmission electron microscopy (TEM) fixative (3\% gluteraldehyde and $1 \%$ paraformaldehyde) after removal of the diseased cornea. Fixation was continued for $48 \mathrm{hr}$ prior to processing for TEM. Five normal corneas were removed within $4 \mathrm{hr}$ of death and processed for TEM by the same technique. The latter corneas could not be used for transplantation due to donor sepsis or other non-ocular exclusion criteria, but were within the age range of 20-45 years of age of the keratoconus corneas used for TEM. Otherwise, TEM was performed as previously described (Michel-Salmin, 1986) using a Joel (Peabody, MA, U.S.A.) Jem 1200E $\times$ electron microscope.

\section{Results}

In 27 out of 45 (60\%) keratoconus corneas, TUNELstained keratocytes were detected in the central region of the corneal button (Fig. 1). Thus, TUNEL-positive keratocytes were detected in a large proportion of corneas with keratoconus (Fig. $1 \mathrm{~A}-\mathrm{C}$, arrowheads). When TUNEL-positive keratocytes were detected they tended to occur in clusters in many of the keratoconus corneas. In each corneal section, several, to as many as 20, stained keratocytes were detected. A large proportion of TUNEL-positive keratocyte cells were near the epithelium in the anterior stroma of keratoconus corneas, and frequently were noted in areas where there was breakdown of Bowman's layer. Some keratoconus corneas had markedly thickened epithelium (Fig. 1C). Fewer TUNEL-stained keratocytes were noted in corneas with stromal dystrophies (Fig. $1 \mathrm{D}$ and $\mathrm{E}$ ) and none were detected in normal corneas (Fig. 1F).

Six out of 17 corneas (35\%) with macular (three out of ten), lattice (two out of four), or granular (one out of three) stromal dystrophy had TUNELstained keratocytes in the central cornea. The difference between the keratococonus and combined stromal dystrophy percentages was statistically significant (Chi-square test, $P=0 \cdot 03$ ).

The two normal, unwounded human corneas (removed for orbital tumor) and four corneas removed within $4 \mathrm{hr}$ of death were also examined for keratocyte apoptosis. No TUNEL-positive keratocytes were detected in more than ten tissue sections from each of these normal corneas. The difference compared to the keratoconus corneas was statistically significant $(P<$ 0·01). TUNEL-positive epithelial cells (arrows) tended 
to only be detectable in the superficial corneal epithelium of normal corneas, whereas in both keratoconus corneas and stromal dystrophy corneas many TUNEL-positive epithelial cells were detected at lower levels in the epithelium.

An analysis of the number of keratocytes detected in each cornea for the keratoconus, stromal dystrophy, and normal corneas was performed. The mean \pm standard error number of TUNEL-positive keratocytes detected in the keratoconus, stromal dystrophy, and normal corneas was $7 \pm 1$ (range $0-20), 2 \pm 0 \cdot 8$ (range $0-9$ ), and $0 \pm 0$ (range $0-0$ ) TUNEL-positive cells per section, respectively. The differences between the keratoconus and the stromal dystrophy $(P=0.0097)$ or the normal cornea $(P=0 \cdot 01)$ groups were statistically significant when evaluated with ANOVA with the Bonferroni/Dunn adjustment. The difference between the stromal dystrophy and normal cornea groups was not statistically significant $(P=0 \cdot 45)$.

Transmission electron microscopy revealed keratocytes that had cellular morphologic features suggestive of apoptosis in the center of the button of each of the keratoconus corneas that were fixed immediately after removal (Fig. 2 A-E). Morphologic changes included cell shrinkage, chromatin condensation, chromatin fragmentation, and membrane-bound bodies budding from cells containing cellular organelles consistent with apoptosis (Wyllie et al., 1980; Arends and Wyllie, 1991). These cell morphologic changes were not detected in the central button of any of the corneas obtained from normal donors (Fig. 2F). Large numbers of small, membrane bound bodies suggestive of apoptotic bodies were noted in the stroma of many keratoconus corneas examined using TEM (Fig. 2 A-E). Statistical analyses are difficult and unreliable with TEM. It was estimated, however, that the proportion of cells with morphologic changes consistent with apoptosis was approximately $1-3 \%$ in each of the keratoconus corneal specimens analysed.

\section{Discussion}

The results of this study suggest that increased keratocyte apoptosis is associated with the corneal ectatic dystrophy keratoconus. The number of specimens per group that were positive for TUNEL-positive keratocytes and the number of TUNEL-positive keratocytes per section were significantly different between the keratoconus and stromal dystrophy or normal cornea groups. Keratocyte apoptosis was detected using the TUNEL assay (Fig. 1). Importantly, we also confirmed the presence of keratocyte apoptosis in keratoconus corneas, but not normal corneas, using transmission electron microscopy (Fig. 2).

Some keratocyte apoptosis that was detected could have been associated with injury to the overlying epithelium that occurred during trephination and removal of the corneal button at the time of penetrating keratoplasty. It is also possible that keratoconus corneas were more susceptible to this trephine-induced apoptosis since they are ectatic. This seems unlikely to be the only source, however, since keratocyte apoptosis in corneas with keratoconus was commonly associated with areas of keratoconusspecific pathology such as damage or disruption of Bowman's layer (Fig. 1). Bowman's damage or disruption tends to be noted in relatively advanced cases of keratoconus. We cannot exclude the possibility that keratocytes are more susceptible to apoptosis due to weakening of the cornea in these areas. However, keratocyte apoptosis was also noted in other areas of the keratoconus corneas. Also, we did not include keratocytes within $500 \mu \mathrm{m}$ of the trephine cut. Our preliminary studies demonstrate that trephine-related keratocyte apoptosis is not detected farther than 200 $\mu \mathrm{m}$ from the cut edge. In addition, the overlying epithelium was intact on each of the corneal sections examined. Finally, if surgery-induced injury was the only source of keratocyte apoptosis in each specimen then the proportion of corneas with keratocyte apoptosis should have been similar in keratoconus and corneas with stromal dystrophies removed using identical techniques. We found keratocyte apoptosis to be present in $60 \%$ of keratoconus corneas and only $35 \%$ of stromal dystrophy corneas. This difference was statistically significant. Keratocyte apoptosis was not detected in any of the normal donor corneas, consistent with our studies in other animals where keratocyte apoptosis is rare in unwounded corneas (Wilson et al., 1996a; Helena et al., 1998). The role of keratocyte apoptosis in the pathogenesis of the other stromal dystrophies used as controls in this study is unknown.

If keratocyte apoptosis contributes to the pathogenesis of keratoconus, then why weren't TUNELpositive keratocytes detected in $100 \%$ of corneas that were removed for keratoconus? There are at least two possible explanations for this finding. First, sampling could have been important. Keratoconus is a disease that typically progresses over a period of time measured in decades and may have periods of progression alternating with periods of remission. Thus, even if more sections from each cornea were examined, it is probable that keratocytes undergoing apoptosis would not be detected in some corneas removed at a particular point in time. Secondly, keratoconus is diagnosed on the basis of clinical findings. Similar to other diseases (hepatitis, for example), there may be several diseases with differing pathophysiological mechanisms that produce the phenotypic change that is currently referred to as keratoconus. Some of these other disorders leading to the keratoconus phenotype may not be associated with keratocyte apoptosis.

How is keratocyte apoptosis that is detected in keratoconus mediated? Our previous studies have suggested that keratocyte apoptosis may be triggered 


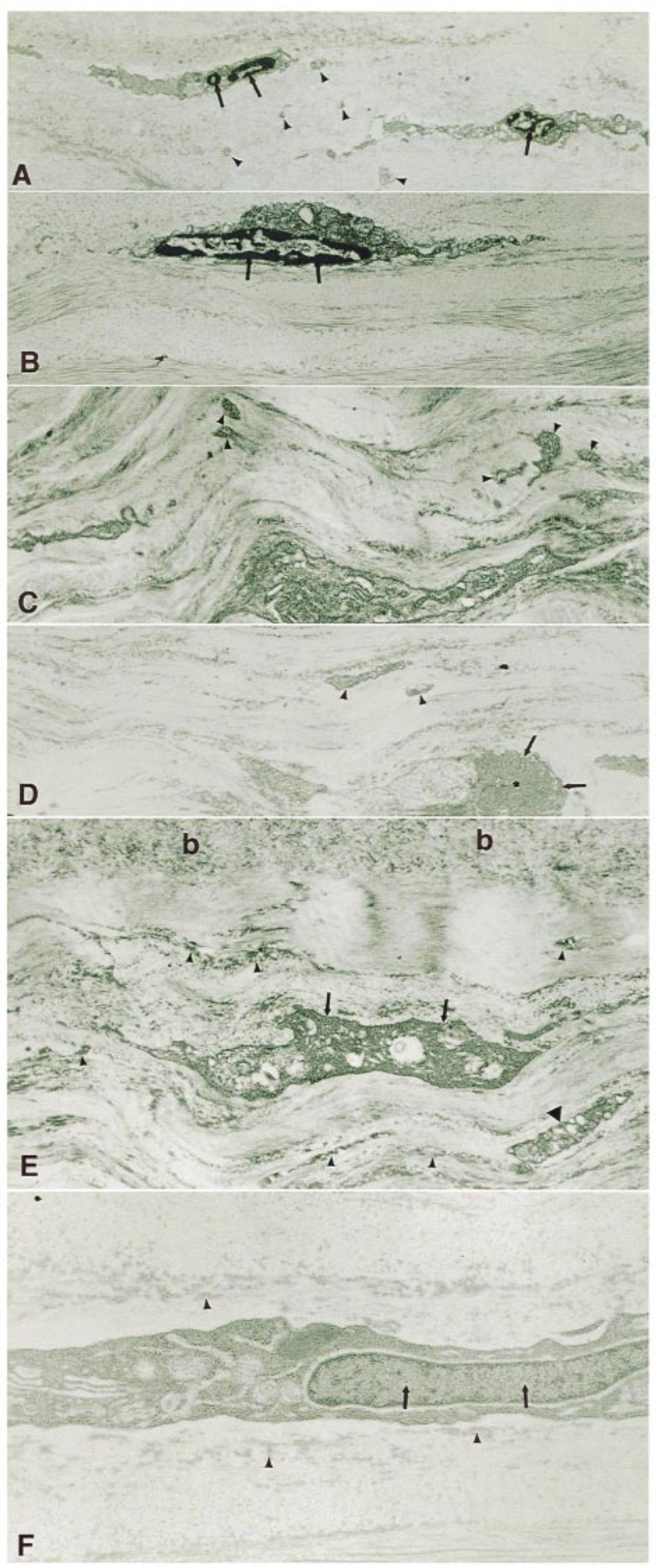

FIG. 2. Transmission electron microscopy of keratoconus and normal corneas. (A) Keratoconus cornea with superficial keratocytes with cell shrinkage, chromatin condensation (arrows) and chromatin fragmentation suggestive of apoptosis. Arrowheads denote membrane bound structures in the surrounding stroma that resemble apoptotic bodies. Magnification $4000 \times$. (B) Keratocyte cell with dense chromatin condensation (arrows), shrinkage, and vacuolization in a cornea with keratoconus. Magnification $6000 \times$. (C) Section from a cornea with keratoconus showing numerous membrane-bound structures (arrowheads) distributed throughout the anterior stroma that resemble apoptotic bodies. Magnification $6000 \times$. (D) Cornea with keratoconus shows a keratocyte with dense chromatin (arrows and asterisk), along with membrane-bound structures in the surrounding stroma that may be apoptotic bodies (arrow- by cytokines such as interleukin-1 (IL-1), Fas ligand, and bone morphogenic protein 2/4 released from the epithelium by injury (Wilson et al., 1996a, 1996b; Helena et al., 1998). This system may serve as a defense mechanism to reduce extension of viruses such as the Herpes simplex virus that infect the corneal epithelium (Wilson et al., 1997). Numerous studies suggest that this system is also activated by mechanical injury to the corneal epithelium (Wilson et al., 1996; Helena et al., 1998). Interestingly, previous studies have associated sources of chronic corneal epithelial injury such as eye rubbing, poorly fit contact lenses, and atopic disease of the eye with keratoconus (Coyle, 1984; Harrison et al., 1989; Koreman, 1986; Krachmer et al., 1984; Macsai et al., 1990; Rahi, 1977). Thus, chronic epithelial injury may link together these seemingly unrelated risk factors associated with keratoconus. Injury-associated increased release of apoptotic cytokines from the epithelium could be an environmental factor associated with the development of keratoconus.

Increased sensitivity of keratocytes to apoptosisinducing cytokines could also be a factor in the pathogenesis of keratoconus. Fabre et al. (1991), and Bureau et al. (1993) noted that corneal tibroblasts from eyes with keratoconus have an average four-fold increase in IL-1 receptors, suggesting that the keratocytes in some or all corneas with keratoconus may have increased sensitivity to IL-1 compared with keratocytes from normal eyes. Previous studies have demonstrated that corneal fibroblasts are stimulated to produce apoptosis-associated Fas ligand in response to IL-1 stimulation (Mohan et al., 1997). Since these cells also express the Fas receptor, abnormal expression of Fas ligand could be associated with autocrine suicide of keratocytes in keratoconus corneas. Abnormalities in other components of these pathways that increase sensitivity to the apoptotic

heads). Magnification $4000 \times$. (E) Bowman's layer can be seen in the top of the panel (b). A degenerating cell can be seen (arrows). This cell does not have typical signs of apoptosis. A large vacuolated cell fragment that appears to contain chromatin and other organelles is also present (large arrowhead). This cell does not have typical signs of apoptosis. A number of smaller electron dense bodies (small arrowheads) indicate a few are present throughout the stroma. Magnification $6000 \times$. (F) Normal donor cornea fixed with TEM within $4 \mathrm{hr}$ of death. No keratocytes with cell morphologic changes consistent with apoptosis were detected in corneas from normal donors. Keratocytes with normal chromatin patterns (arrows) were detected throughout the stroma of the donor corneas. Intact organelles such as mitochondria and endoplasmic reticulum can be seen in the cytoplasm of the normal cell. Nondescript electron dense structures could be detected in some areas of normal corneas (arrowheads). Membrane bound structures suggestive of apoptotic bodies were rarely seen. Magnification $6000 \times$. 
cytokines could also underlie the development of keratoconus.

Apoptosis, in contrast to necrosis, is a special form of cell death that occurs with minimal release of degradative enzymes and other components that would damage surrounding cells (Wyllie et al., 1980; Arends and Wyllie, 1991; Wilson et al., 1996b). How could this process underlie the development of an ectatic corneal disease associated with stromal thinning and disruption of Bowman's layer? One possibility may be that apoptosis is imperfect and some intracellular components escape into the surrounding tissue. In an acute situation of an infection or mechanical scrape of the epithelium, the escape of limited amounts of degradative enzymes is not likely to be significant, since enzyme inhibitors and other regulatory components are also present in the tissue. However, ongoing release of small amounts of these degradative enzymes over an extended period of time could result in tissue damage. Several studies have detected degradative enzymes and other components at increased levels in keratoconus corneas (Kao et al., 1982; Sawaguchi et al., 1989; Fini et al., 1992; Fukuchi et al., 1994; Kenny et al., 1994; Smith et al., 1995). Chronic apoptosis could be the factor that links together these observations of increased levels of differing degradative components produced by keratocytes.

Further studies are needed to confirm the association between keratocyte apoptosis and keratoconus. Genetic factors associated with keratocyte apoptosis and keratoconus should be investigated, especially as they relate to regulation of the keratocyte apoptosis response. Confirmation of the role of keratocyte apoptosis in the pathophysiology of this disease could lead to new therapeutic strategies to prevent progression of the keratoconus once it is detected in its early stages.

\section{Acknowledgments}

Supported by US Public Health Service grants EY10056 (SEW) and EY09052 (YSR) from the National Eye Institute, Bethesda, Maryland, an unrestricted grant from Research to Prevent Blindness, New York, NY (SEW), and The Eye Birth Defects Research Foundation, Inc. (YSR).

\section{References}

Arends, M. J. and Wyllie, A. H. (1991). Apoptosis: mechanisms and roles in pathology. Int. Rev. Exp. Pathol. 32, 223-54.

Bereau, J., Fabre, E. J., Hecquet, C., Pouliquen, Y. and Lorans, G. (1993). Modification of prostiglandin E2 and collagen synthesis in keratoconus fibroblasts associated with an increase of interleukin-1 alpha receptor number. C. R. Acad. Sci. III. 316, 425-30.

Campos, M., Szerenyi, K., Lee, M., McDonnell, J. M. and McDonnell, P. J. (1994). Keratocyte loss after corneal deepithelialization in primates and rabbits. Arch. Ophthalmol. 112, 254-60.

Coyle, J. T. (1984). Keratoconus and eye rubbing. Am. J. Ophthalmol. 97, 527-8.

Fabre, E. J., Bureau, J., Pouliquen, Y. and Lorans, G. (1991). Binding sites for human interleukin-1 alpha, gamma interferon and tumor necrosis factor on cultured fibroblasts of normal cornea and keratoconus. Curr. Eye Res. 10, 585-92.

Fini, M. E., Yue, B. Y. and Sugar, J. (1992). Collagenolytic/gelatinolytic metalloproteinases in normal and keratoconus corneas. Curr. Eye Res. 11, 849-62.

Fukuchi, T., Yue, B. Y., Sugar, J. and Lam, S. (1994). Lysosomal enzyme activities in conjunctival tissues of patients with keratoconus. Arch. Ophthalmol. 112, $1368-74$.

Harrison, R. J., Klouda, P. T., Easty, D. L., Manku, M., Charles, J. and Stewart, C. M. (1989). Association between keratoconus and atopy. Br. J. Ophthalmol. 73, 816-22.

Helena, M. C., Baerveldt, F., Kim, W-J. and Wilson, S. E. (1998). Keratocyte apoptosis following corneal surgery. Invest. Ophthalmol. Vis. Sci. 39, 276-83.

Kao, W. W., Vergnes, J. P., Ebert, J., Sundar-Raj, C. V. and Brown, S. I. (1982). Increased collagenase and gelatinase activities in keratoconus. Biochem. Biophys. Res. Commun. 107, 929-36.

Kennedy, R. H., Bourne, W. M. and Dyer, J. A. (1986). A 48year clinical and epidemiological study of keratoconus. Am. J. Ophthalmol. 101, 267-73.

Kenney, M. C., Chwa, M., Opbroek, A. J. and Brown, D. J. (1994). Increased glatinolytic activity in keratoconus keratocyte cultures. A correlation to an altered matrix metalloproteinase-2/tissue inhibitor of metalloproteinase ratio. Cornea. 13, 114-24.

Koreman, N. M. (1986). A clinical study of contact-lensrelated keratoconus. Am. J. Ophthalmol. 101, 390-92.

Krachmer, J. H., Felder, R. S. and Belin, M. W. (1984). Keratoconus and related noninflammatory corneal thinning disorders. Surv. Ophthalmol. 28, 293-322.

Macsai, M. S., Varley, G. A. and Krachmer, J. H. (1990). Development of keratoconus after contact lens wear. Arch. Ophthalmol. 108, 534-8.

Michel-Salmin, L. A., Tosi-Couture, E., Gautier, A., McDowall, A. W. and Dubochet, J. (1986). Electron microscopy of the chromosomes of Dinoflagellate Porocentrum micans: confirmation of Bouligandíss liquid crystal hypothesis. J. Ultrastruct. Molec. Struct. Res. 97, 10-30.

Mohan, R. R., Kim, W-J., Mohan, R. R., Chen, L. and Wilson, S. E. (in press). Bone morphogenic proteins 2 and 4 and their receptors in the adult human cornea. Invest. Ophthalmol. Vis. Sci.

Mohan, R. R., Liang, Q., Kim, W-J., Helena, M. C., Baerveldt, F. and Wilson, S. E. (1997). Apoptosis in the cornea: further characterization of Fas/Fas ligand system. Exp. Eye Res. 65, 575-89.

Rabinowitz, Y. S. (1998). Keratoconus. Surv. Ophthalmol. 42, 297-319.

Rahi, A., Davies, P., Ruben, M., Lobascher, D. and Menon, J. (1977). Keratoconus and coexisting atopic disease. Br. J. Ophthalmol. 61, 761-4.

Sawaguchi, S., Yue, B. Y., Sugar, J. and Gilboy, J. E. (1989). Lysosomal enzyme abnormalities in keratoconus. Arch. Ophthalmol. 107, 1507-10.

Smith, V. A., Hoh, H. B., Littleton, M. and Easty, D. L. (1995). Over-expression of a gelatinase A activity in keratoconus. Eye 9, 429-33.

Wilson, S. E., He, Y-G., Weng, J., Li, Q., Vital, M. and Chwang, E. L. (1996a). Epithelial injury induces kerato- 
cyte apoptosis: hypothesized role for the interleukin-1 system in the modulation of corneal tissue organization. Exp. Eye Res. 62, 325-38.

Wilson, S. E. and Kim, W-J. (1998). Keratocyte apoptosis: implications on corneal wound healing, tissue organization, and disease. Invest. Ophthalmol. Vis. Sci. 39, 220-6.

Wilson, S. E. and Klyce, S. D. (1991). The topography of keratoconus. Cornea 10, 2-8.

Wilson, S. E., Li, Q., Weng, J., Barry-Lane, P. A., Jester, J. V.,
Liang, Q. and Wordinger, R. J. (1996b). The Fas/Fas ligand system and other modulators of apoptosis in the cornea. Invest. Ophthalmol. Vis. Sci. 37, 1582-92.

Wilson, S. E., Pedroza, L., Beuerman, R. and Hill, J. (1997). Herpes simplex virus type-1 infection of corneal epithelial cells induces apoptosis of the underlying keratocytes. Exp. Eye Res. 64, 775-9.

Wyllie, A. H., Kerr, J. F. and Currie, A. R. (1980). Cell death: the significance of apoptosis. Int. Rev. Cytol. 68, 251-306. 